\title{
Use of an Extra-Tympanic Membrane Electrode to Record Cochlear Microphonics with Click, Tone Burst and Chirp Stimuli
}

\author{
Laura M. Coraci and Andy J. Beynon *(1)
}

check for

updates

Citation: Coraci, L.M.; Beynon, A.J. Use of an Extra-Tympanic Membrane Electrode to Record Cochlear

Microphonics with Click, Tone Burst and Chirp Stimuli. Audiol. Res. 2021, 11, 89-99. https://doi.org/10.3390/ audiolres11010010

Received: 7 January 2021

Accepted: 22 February 2021

Published: 1 March 2021

Publisher's Note: MDPI stays neutral with regard to jurisdictional claims in published maps and institutional affiliations.

Copyright: (c) 2021 by the authors. Licensee MDPI, Basel, Switzerland. This article is an open access article distributed under the terms and conditions of the Creative Commons Attribution (CC BY) license (https:/ / creativecommons.org/licenses/by/ $4.0 /)$.
Vestibular \& Auditory EP Lab-Department Otorhinolaryngology, Radboud University Medical Center, Ph. Van Leijdenlaan 15, 6525EX Nijmegen, The Netherlands; lauramc@live.nl

* Correspondence: andy.beynon@radboudumc.nl

\begin{abstract}
This study determined electrocochleography (ECochG) parameter settings to obtain cochlear microphonics (CM) with less invasive flexible extra-tympanic membrane electrodes. In 24 adult normal-hearing subjects, CMs were elicited by presenting click stimuli at $100 \mathrm{dBnHL}$, tone bursts $(2 \mathrm{kHz})$ and broadband (BB) CE-chirps ${ }^{\circledR}$ LS (Interacoustics, Middelfart, Denmark), both at $80 \mathrm{dBnHL}$. Different high-pass filters (HPFs) (3.3 Hz and $100 \mathrm{~Hz}$, respectively) were used to investigate response quality of the CM. CMs were successfully obtained in 92-100\% with click-, 75-83\% with $2 \mathrm{kHz}$ tone burst- and 58-63\% with CE-chirp ${ }^{\circledR}$-LS stimuli. Click stimuli elicited significantly larger CM amplitudes compared to $2 \mathrm{kHz}$ tone bursts and BB CE-chirp ${ }^{\circledR}$ LS (Interacoustics, Middelfart, Denmark). No significant differences were found between the two different high-pass filter (HPF) settings. The present study shows that it is possible to obtain clear CMs with the flexible extratympanic membrane electrodes using click stimuli. In contrast to $2 \mathrm{kHz}$ tone bursts and CE-chirp ${ }^{\circledR}$ (Interacoustics, Middelfart, Denmark) LS, clicks show a significantly higher success rate and are the preferred stimuli to confirm the presence or absence of CMs.
\end{abstract}

Keywords: electrocochleography; cochlear microphonic; tympanic membrane electrode; auditory evoked potentials

\section{Introduction}

Auditory evoked potentials (AEPs) from the cochlea and the auditory nerve can be assessed objectively by electrocochleography (ECochG), revealing four basic components within the first $5 \mathrm{~ms}$ after stimulus onset: (1) the compound action potential (AP), which can be described as a reflection of the combined firing of thousands of cochlear nerve fibers and can be clinically interpreted as a measure of the actual auditory response or "hearing potential"; (2) the summating potential (SP), which reflects the nonlinear distortion from the outer hair cells (OHCs) [1]; (3) the auditory nerve neurophonic (ANN), reflecting the auditory nerve firing most likely to occur as a response to low frequency tones [2]; and (4) the cochlear microphonic (CM), which is a preneural reproduction of the acoustic signal that "mirrors" the movement of the basilar membrane, reflecting the spatial summation of transducer currents produced by a large number of OHCs [3]. The first ECochG measurements in humans during surgery were performed by Perlman and Case in 1941 [4]. In the sixties, the development of computer averaging algorithms enabled the first nonsurgical ECochG recordings under local anesthesia [5]. This transtympanic (TT) ECochG is a rather invasive procedure, requiring an insertion and middle-ear placement of a needle recording electrode through the tympanic membrane, usually carried out by a surgeon. However, in the eighties, the less invasive Brainstem Evoked Response Audiometry (BERA) became more popular in clinical audiology to objectively assess hearing thresholds since it covered a wider range of the auditory pathway from the cochlear nerve (including the AP) up to the level of the auditory brainstem. Although, 
for the diagnosis, evaluation or prognosis of hearing losses in specific patient groups, such as Meniere's Disease (calculating the ratio between the SP and compound AP as an indicator for endolymphatic hydrops), ECochG was still applied [6].

Besides the fact that CMs, in contrast to compound APs, appeared to be not very useful in assessing hearing thresholds and were also more difficult to distinguish from artefacts, its clinical value was rather limited [1]. Nevertheless, during the last decades, the CM has been gaining more clinical interest in the assessment of specific auditory pathologies; it is suggested that this preneural response could be of importance in patient groups where one is specifically interested in the functioning of different cochlear structures, such as in patients with mitochondrial hearing disorders [7]. Other ECochG studies reported its application in patients with auditory neuropathy/dyssynchrony spectrum disorders (ANSD). Since ANSD is characterized by normal OHC function but with absent or a disturbed neural synchrony at the higher brainstem level, subjects with ANSD reveal abnormally increased CM amplitudes during ECochG in comparison to normal-hearing subjects. Thus, advocating for the application of $\mathrm{CM}$ recordings as part of the diagnostic protocol to confirm ANSD should be considered [8].

More recently, $\mathrm{CM}$ recordings seem to be promising for the assessment of intracochlear trauma of the OHCs during the insertion of a cochlear implant [9]. These recent developments contributed to a revival of ECochG applications in clinical settings. For a systematic review, see Trecca et al. [10].

Although both otoacoustic emissions (OAEs) and CMs assess OHC activity, the lower frequencies (e.g., $500 \mathrm{~Hz}$ ) are more difficult to obtain with OAEs compared to CMs, suggesting that CMs may have a higher diagnostic value [11].

To date, most studies are using a relatively invasive transtympanic (TT)-ECochG setup by inserting the electrode through the tympanic membrane and placing it on the cochlear round window or promontory to obtain $\mathrm{CMs}$ for $\mathrm{OHC}$ diagnosing purposes [12]. An alternative to this invasive TT-ECochG is the application of an extra-tympanic (ET) recording electrode placement in the ear canal or near the tympanic membrane. Even though the response amplitudes of ET-ECochG are four times smaller compared to TT-ECochG responses due to the greater distance to the neural source [13], ET-ECochG is still preferred in clinical audiology due to its less invasive and more patient-friendly nature. Nowadays, most ET-ECochG responses are captured by using a "tiptrode", an "eartrode" or a tympanic membrane (TM) electrode. "Tiptrodes" are insert phone plugs that are wrapped in gold foil, the latter functioning as a recording site, while "eartrodes" are placed in middle part of the ear canal. A TM electrode is a flexible silicon-shielded electrode that can be placed closer to, or even against, the tympanic membrane, thus closer to the source of the neural response. A within-subject experiment comparison of those two ET methods reported a significant difference between the two electrode positions: ECochG with a TM electrode (from now on called TM-ECochG) revealed significantly higher response amplitudes and better reproducibility due to its closer proximity to the cochlea compared to "tiptrodes" located in the ear canal [13]. However, in contrast to "tiptrodes", placement of a TM electrode requires some practical skills of the clinician to carefully place the electrode as close as possible to the tympanic membrane.

The quality of ECochG recordings, i.e., peak amplitudes and latencies, is highly dependent on specific recording parameters (electrode positions, electrode brand and filter settings) and stimulation parameters (stimulus type, stimulus repetition rate and polarity). With respect to the ECochG parameter setting, there seems to be no common consensus on the specific frequency for the high-pass filter (HPF). A wide variety of HPFs are clinically used, varying from $5[14,15]$ to $100 \mathrm{~Hz}[16,17]$. Since recording and acquisition parameters vary between different clinics, it is therefore recommended to obtain normative ECochG values based on their local clinic-specific recording conditions [18].

The current study will investigate the clinical feasibility to use a flexible ET silicon TM electrode to acquire CMs in normal-hearing subjects, as (1) the TM-ECochG recording is less invasive compared to the TT variant, and (2) it leads to higher response peak-to-peak 
amplitudes compared to the application of the ET alternative, "tiptrode" recordings [13]. In contrast to the few previous TM-ECochG studies, mainly focused on just AP and SP recordings [19], this study will specifically focus on the acquisition of CM responses. Since chirp stimuli have been clinically implemented in clinical AEP recording systems in the last decade [20], our second aim is to investigate which of the different stimulus types, including the level specific chirp (CE-chirp ${ }^{\circledR}$ LS) (Interacoustics, Middelfart, Denmark), is preferred for optimal $\mathrm{CM}$ response recordings.

\section{Materials and Methods}

\subsection{Subjects}

Twenty-four normal-hearing adult subjects (10 males, 14 females) with a mean age of 24.6 years \pm 2.6 (range: $20-32$ years) participated in this study. All participants had pure tone thresholds $\leq 20 \mathrm{dBnHL}$ from 250 to $8000 \mathrm{~Hz}$ for both ears and did not have any oto-neurological history. All subjects read the information brochure and signed the informed consent before participation. Participation was completely voluntarily.

\subsection{TM-ECochG Parameters}

An AEP recording system (Interacoustics Eclipse II ${ }^{\circledR}$, Denmark) was used to measure the right ear of each subject. The impedances of all disposable surface electrodes were $\leq 5 \mathrm{k} \Omega$. A recording-time window of $10 \mathrm{~ms}$ in length was used to capture and average the ECochG responses. Considering the stimulus travel time through the $26.6 \mathrm{~cm}$ silicon tube, all stimuli arrived at the tympanic membrane at exactly $0 \mathrm{~ms}$. The system has an integrated digital filter, and the responses were preamplified 100,000 times.

Three different test protocols were used to obtain CMs in response to a click, $2 \mathrm{kHz}$ tone burst and broad band (BB) CE-chirp ${ }^{\circledR}$ LS. To facilitate a clear interpretation of consistent $\mathrm{CM}$ responses, all stimuli were presented at a loud acceptable presentation level (LAPL) stimulus intensity of $100 \mathrm{dBnHL}$ (click, $0.1 \mathrm{~ms}$ ) and $80 \mathrm{dBnHL}$ ( $2 \mathrm{kHz} 1-1-1$ cycle tone burst and CE-chirp ${ }^{\circledR}$ LS), respectively, after subjective equal loudness perception was confirmed for all three stimuli in a (pilot) loudness scaling experiment at chosen stimulation levels. A stimulus repetition rate of $87.1 / \mathrm{s}$ was used with two different high-pass filters (HPFs), i.e., 3.3 or $100 \mathrm{~Hz}$, and a fixed low-pass filter of $3000 \mathrm{~Hz}$. To confirm reproducibility, all TMECoch G responses were recorded at least twice for each stimulus polarity and averaged for all condensation and rarefaction responses. According to current CM protocols [17], CMs were confirmed by two additional recordings with a clamped tube, confirming absent $\mathrm{CM}$ responses. For each recording, 1500 stimuli were presented for each stimulus type consisting of at least six recordings: at least 2 condensation and rarefaction responses each and 2 clamped conditions with a rarefaction polarity (arbitrarily chosen) were recorded with an HPF of $3.3 \mathrm{~Hz}$. The same procedure was repeated with an HPF set at $100 \mathrm{~Hz}$.

\subsection{Procedure}

Prior to the recordings, the right ear canal of each subject was inspected for outer ear and/or tympanic abnormalities and cleaned (cerumen), followed by conventional tonal audiometry to confirm normal hearing. Fz and Fpz scalp locations were prepared with chlorhexidine $0.5 \%$ in ethanol $70 \%$ and scrubbed with a mild abrasive gel to reduce skin impedance. Subsequently, disposable surface electrodes were covered with conductive electrode paste and placed on Fz (inverting electrode) and on middle-forehead/Fpz (ground). Tables 1 and 2 summarize stimulus and recordings parameters. 
Table 1. Stimulus parameters for the CM protocols per stimulus type (click, $2 \mathrm{kHz}$ tone burst and CE-chirp ${ }^{\circledR}$ LS).

\begin{tabular}{cccc}
\hline Stimulus Parameters & Protocol 1 & Protocol 2 & Protocol 3 \\
\hline Stimulus type & Click & $2 \mathrm{kHz}$ tone burst & ${ }^{1}$ BB CE-chirp ${ }^{\circledR}$ LS \\
Stimulus rate $(\mathrm{Hz})$ & 87.1 & 87.1 & 87.1 \\
Polarity & 2 Rare \& ${ }^{3}$ cond & Rare \& cond & Rare \& cond \\
Duration (ms) & 0.1 & 1.5 & 5 \\
Envelope & - & $1-1-1$ cycle & - \\
Intensity (dBnHL) & 100 & 80 & 80 \\
\hline
\end{tabular}

${ }^{1} \mathrm{BB}=$ broadband ${ }^{2}$ rare $=$ rarefaction,${ }^{3}$ cond $=$ condensation.

Table 2. Recording parameters for all CM protocols.

\begin{tabular}{cc}
\hline Recording Parameter & CM Protocols \\
\hline Stimulation & Monaural \\
Type electrode & Tympanic membrane (TM) electrode (Sanibel, Denmark) \\
Electrode positioning & Vertical montage \\
Averaging (total) & $2 \times 1500(3000)$ \\
${ }^{1}$ HPF-2 LPF (Hz) & $3.3-3000$ or 100-3000 \\
Amplification & $100,000 \times$ \\
\hline
\end{tabular}

${ }^{1} \mathrm{HPF}=$ high-pass filter, ${ }^{2} \mathrm{LPF}=$ low-pass filter.

The TM electrode (Sanibel, Denmark) was allocated in the AEP electrode interface (EPA4, Interacoustics) as inverting, according to standard "vertical montage" and connected to the jumper between left and right to allow single channel recordings (see Figure 1).

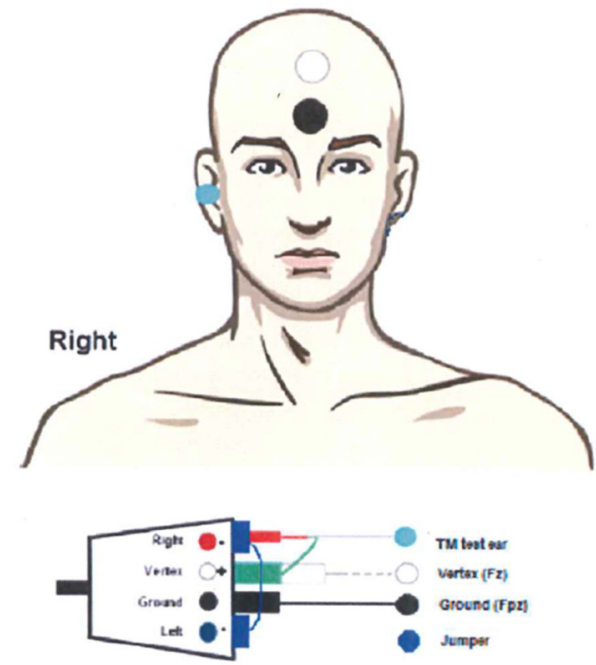

Figure 1. Electrode configuration using the TM electrode and EPA4 (with permission of Interacoustics, 2019).

The patient was instructed to lie down comfortably on a bed on the left side (right ear up). To avoid any discomfort during electrode insertion, a puff of $10 \%$ xylocaine was sprayed in the right ear canal. In the meantime, the TM electrode was placed in a bath of saline and conductivity gel (Lectron II) with ratio 1:1 at room temperature for $10 \mathrm{~min}$.

Then, the ear canal was dried with a cotton bud, followed by TM-electrode placement at the superior half of the tympanic membrane, until low resistance was noticed. Lastly, an insert probe (E-A-RTONE 5A) was placed in the ear canal, simultaneously fixating the TM electrode (see Figure 2). Depending on the size of the individual ear canal, foam was cut approximately $2 \mathrm{~mm}$ around the tip, avoiding any obstruction or leakage of the soundwave. The subjects were instructed to lie down relaxed. When ECochG recordings were completed, 
a final ear canal inspection and cleansing of the ear canal were carried out. The whole experiment was performed in a soundproof and light-dimmed room and took about $75 \mathrm{~min}$ in total, including preparation time.

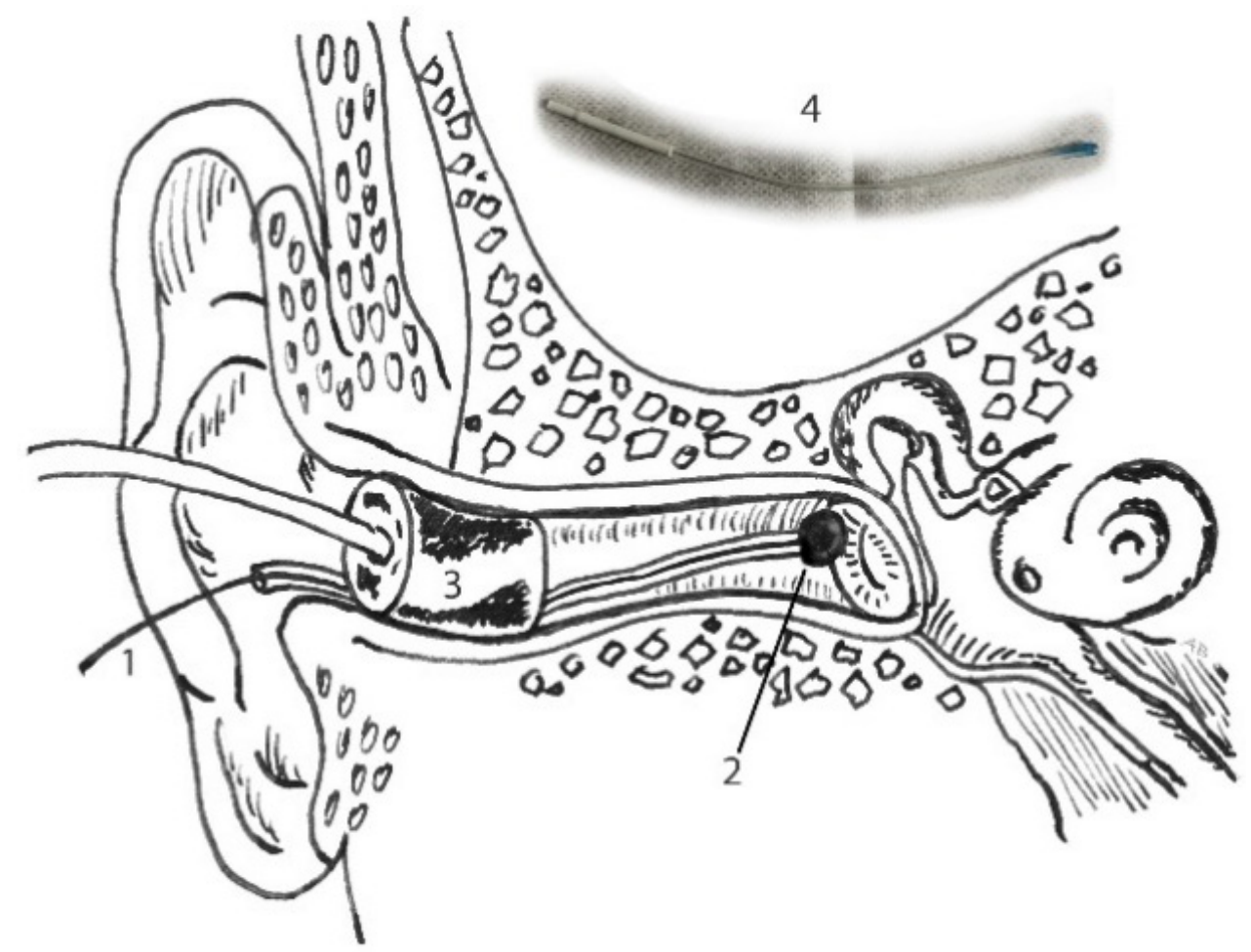

Figure 2. Placement of shielded tympanic membrane (TM) electrode with gel tip, fixed by placement of the insert probe: 1. Lead TM electrode (recording), 2. gel tip, directly placed against the superior half of the tympanic membrane, 3 . insert probe (stimulus) and 4. inset: TM electrode (Sanibel ${ }^{\mathrm{TM}}$ ).

\subsection{Statistical Analysis}

Overall prevalence of the $\mathrm{CM}$ waveforms was calculated. $\mathrm{CM}$ was considered present when the responses from both polarities show an inversed phase, with an absence of the responses in the clamped-tube condition. The $\mathrm{CM}$ was defined as absent when both polarities were not reversible or reproducible or when a response was seen in the clamped tube condition.

Analysis using IBM SPSS Statistics version 21 (IBM SPSS, Inc., Chicago, IL, USA) was conducted to provide descriptive statistics for analysis of the success rate and means per stimulus type and HPF. Polarities were described individually. A repeated measures ANOVA design with planned contrasts was executed to analyze the difference in $\mathrm{CM}$ amplitude, CM latency and CM duration after changing the stimulus types (click, $2 \mathrm{kHz}$ tone burst and CE-chirp ${ }^{\circledR}$ LS) and HPF $(3.3 \mathrm{~Hz}$ and $100 \mathrm{~Hz})$. Levels of significance were defined at the $5 \%$ level $(p<0.05)$. Peak-to-peak amplitudes of CM sinusoidal response $(\mathrm{nV})$ were used for analyses: for clicks and CE-chirps ${ }^{\circledR}$ LS, amplitude was at the onset of first response; and for $2 \mathrm{kHz}$, amplitude was of the second sinusoid (1-1-1). CM latencies were defined as the time from the onset of the stimulus to the first $\mathrm{CM}$ response in milliseconds. $\mathrm{CM}$ durations were calculated from the onset of the first $\mathrm{CM}$ sinusoid until the end of the last CM sinusoid. Data was corrected when assumptions of normality and sphericity were violated. Differences between sexes and polarities were explored with one-way ANOVAs. 


\section{Results}

\subsection{Success Rates in Response to the Different Stimuli}

TM-ECochG recordings of responses to acoustic stimuli were obtained in 24 normalhearing adults. CMs were recognized in the recorded ECochG in response to a click stimulus (92-100\%), a $2 \mathrm{kHz}$ tone burst $(75-83 \%)$ and a CE-chirp ${ }^{\circledR}$ LS (58-63\%): see Table 3.

Table 3. Success rates of cochlear microphonics (CM) in response to each stimulus type.

\begin{tabular}{ccccc}
\hline${ }^{1}$ HPF & ${ }^{2}$ LPF & CM Click & $\begin{array}{c}\text { CM 2 kHz } \\
\text { Tone Burst }\end{array}$ & CM CE-Chirp ${ }^{\circledR}$ LS \\
\hline $3.3 \mathrm{~Hz}$ & $3000 \mathrm{~Hz}$ & $22 / 24(92 \%)$ & $20 / 24(83 \%)$ & $14 / 24(58 \%)$ \\
\hline $100 \mathrm{~Hz}$ & $3000 \mathrm{~Hz}$ & $24 / 24(100 \%)$ & $18 / 24(75 \%)$ & $15 / 24(63 \%)$ \\
\hline
\end{tabular}

${ }^{1} \mathrm{HPF}=$ high-pass filter, ${ }^{2} \mathrm{LPF}=$ low-pass filter.

Typically, all subjects show clear CM responses evoked by a click: see Figure 3, left graph. An example of a subject with one of the best CM responses evoked by both sinusoidal stimuli (atypical) is shown in Figure 3 (middle and right graph). In most of the subjects, CM responses to tone bursts and CE-chirp ${ }^{\circledR}$ LS were worse or hardly recognizable; i.e., they showed very low amplitudes.
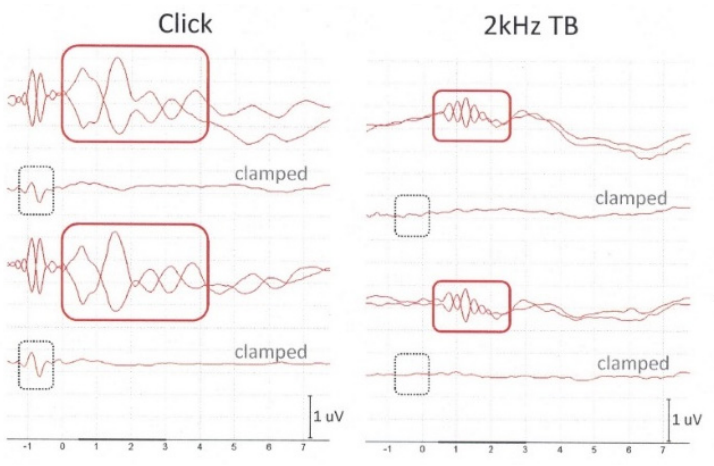

BB-CE chirp

Figure 3. Example of a single subject showing from top to bottom: CMs (both polarities superimposed, 3.3 Hz HPF), the clamped tube condition (no CM), CMs (both polarities superimposed, $300 \mathrm{~Hz} \mathrm{HPF),}$ clamped tube condition (no CM) evoked by a click, typical for all subjects (left graph), by a tone burst (atypical response: middle graph) and by a BB-CE chirp stimulus (atypical response: right graph). Note: in contrast to conventional lower stimulation rates used for SP/AP recordings, a higher stimulation rate was used to obtain CMs $(87.1 \mathrm{~Hz})$. Black rectangles: residual electrical stimulus artifact; red rectangle: $\mathrm{CM}$ response.

\subsection{Different Parameter Settings}

Since normality of the sample mean distributions were violated (Shapiro-Wilk $p<0.05$ ) and the assumption of sphericity was violated, data was corrected first, using GreenhouseGeisser $(\varepsilon<0.75)$ and Shapiro-Wilk.

A repeated measures ANOVA design was applied to analyze $\mathrm{CM}$ response differences with different parameter settings. A significant main effect for stimulus $(\mathrm{F}(1.01,9.12)=28.71$, $\left.p<0.001, \eta^{2} \mathrm{p}=0.76\right)$ and a significant interaction effect for stimulus and polarity $(\mathrm{F}(1.04$, 9.36) $\left.=25.87, p<0.001, \eta^{2} p=0.74\right)$, both corrected with Greenhouse-Geisser, were found. Planned contrasts revealed that the click stimulus evoked a significantly larger CM amplitude than the $2 \mathrm{kHz}$ tone burst stimulus $\left(\mathrm{F}(1,9)=28.49, p<0.001, \eta^{2} \mathrm{p}=0.76\right)$ and the CE-chirp ${ }^{\circledR}$ LS stimulus $\left(\mathrm{F}(1,9)=24.41, p<0.001, \eta^{2} \mathrm{p}=0.73\right)$. CM amplitudes evoked by the $2 \mathrm{kHz}$ tone burst stimulus and the CE-chirp ${ }^{\circledR}$ LS stimulus did not differ significantly $\left(\mathrm{F}(1,9)=0.001, p>0.05, \eta^{2} \mathrm{p}=0.00\right)$. Mean CM amplitudes are shown in Table 4. 
Table 4. Means and standard deviations of CM amplitudes (in $\mathrm{nV}$ ) evoked by click, $2 \mathrm{kHz}$ tone burst and CE-chirp ${ }^{\circledR}$ LS.

\begin{tabular}{ccccc}
\hline Stimulus Type & Polarity & $\mathbf{1}^{\mathbf{H P F}}$ & $\begin{array}{c}\text { Females } \\
\text { CM Amplitude } \\
\text { Mean (4) SD) }\end{array}$ & $\begin{array}{c}\text { Males } \\
\text { CM Amplitude } \\
\text { Mean (SD) }\end{array}$ \\
\hline \multirow{2}{*}{ Click } & 2 Cond & $100 \mathrm{~Hz}$ & $1.37(0.93)$ & $2.57(1.15)$ \\
& ${ }^{3}$ Rare & $3.3 \mathrm{~Hz}$ & $1.00(0.67)$ & $1.68(0.95)$ \\
& & $100 \mathrm{~Hz}$ & $0.94(0.60)$ & $1.67(0.82)$ \\
$2 \mathrm{kHz}$ tone burst & Cond & $100 \mathrm{~Hz}$ & $0.12(0.05)$ & $0.13(0.04)$ \\
& Rare & $3.3 \mathrm{~Hz}$ & $0.15(0.07)$ & $0.11(0.03)$ \\
& & $100 \mathrm{~Hz}$ & $0.14(0.05)$ & $0.12(0.04)$ \\
& & $3.3 \mathrm{~Hz}$ & $0.23(0.12)$ & $0.12(0.02)$ \\
\hline \multirow{3}{*}{ CE-chirp ${ }^{\circledR} \mathrm{LS}$} & Cond & $100 \mathrm{~Hz}$ & $0.20(0.11)$ & $0.12(0.08)$ \\
& Rare & $3.3 \mathrm{~Hz}$ & $0.21(0.11)$ & $0.16(0.08)$ \\
& & $100 \mathrm{~Hz}$ & $0.21(0.10)$ & $0.19(0.06)$ \\
\hline
\end{tabular}

${ }^{1} \mathrm{HPF}=$ high-pass filter,${ }^{2}$ cond $=$ condensation, ${ }^{3}$ rare $=$ rarefaction, ${ }^{4} \mathrm{SD}=$ standard deviation.

The use of different HPFs, analyzed by repeated measures ANOVA, showed no significant different $\mathrm{CM}$ responses $\left(\mathrm{F}(1.01,9.17)=2.79, p<0.05, \eta^{2} \mathrm{p}=0.24\right)$ for all three stimuli.

One-way ANOVAs were executed and showed significant differences between sexes for CM amplitudes evoked by a click and $3.3 \mathrm{~Hz} \mathrm{HPF}$ in condensation (males $=2.57 \mathrm{nV}$, females $=1.37 \mathrm{nV}, \Delta=1.20 \mathrm{nV}, p<0.05$ ) and rarefaction polarity (males $=1.67 \mathrm{nV}$, females $=1.0 \mathrm{nV}, \Delta=0.67 \mathrm{nV}, p<0.05)$. The absolute means per sexes are seen for each condition in Table 4 . With respect to the other two stimuli, CM amplitudes evoked by $2 \mathrm{kHz}$ tone bursts and CE-chirp ${ }^{\circledR}$ LS were similar for both sexes; no significant differences were found $(p>0.05)$. Although not statistically significant, CM amplitudes evoked by a click stimulus showed larger amplitudes for both polarities when HPF was changed to $100 \mathrm{~Hz}$. The use of different HPFs had larger consequences for the CM amplitudes evoked by click stimuli of males (largest $\mathrm{SD}=1.15 \mathrm{nV}$ ) than females (largest $\mathrm{SD}=0.93 \mathrm{nV}$ ), assessed by a one-way ANOVA $(p<0.05)$.

\subsection{Latency and Duration}

The planned contrasts showed no significant interactions between CM latency and CM duration when stimulus type or $\operatorname{HPF}$ was changed: $\mathrm{CM}$ latency $(\mathrm{F}(2,18)=0.39, p>0.05$, $\left.\eta^{2} p=0.04\right)$ and $C M$ duration $\left(F(1.12,10.11)=4.13, p>0.05, \eta^{2} p=0.31\right)$ (repeated measures ANOVA).

With respect to differences between sexes, one-way ANOVAs showed no significant differences between the sexes for CM duration $(p>0.05)$, but a significant difference was seen for the CM latency evoked by a CE-chirp ${ }^{\circledR}$ LS with HPF setting $100 \mathrm{~Hz}$ in both polarities, i.e., condensation (males $=0.82 \mathrm{~ms}$, females $=0.43 \mathrm{~ms}, \Delta=0.56 \mathrm{~ms}, p<0.05$ ) and rarefaction (males $=1.06 \mathrm{~ms}$, females $=0.52 \mathrm{~ms}, \Delta=0.54 \mathrm{~ms}, p<0.05$ ).

\section{Discussion}

The purpose of this study was to investigate the feasibility of using flexible silicon ET membrane electrodes instead of relatively invasive TT needle electrodes to obtain CMs. To our knowledge, this is the first study that explicitly describes the recording of CMs obtained with a flexible silicon TM electrode comparing clicks, $2 \mathrm{kHz}$ tone bursts and CE-chirp ${ }^{\circledR}$ LS stimuli. In addition to analyzing and proposing the most optimal stimulus parameter settings with respect to the different stimulus types, the HPF recording parameter was also analyzed for two different HPF settings during CM acquisition.

Firstly, results show that it is possible to obtain CMs with flexible silicon noninvasive TM electrodes. Secondly, the ECochG results reveal a $92 \%-100 \%$ presence of CMs when evoked by click stimuli, followed by a success rate of $75 \%-83 \%$ in response to $2 \mathrm{kHz}$ 
tone bursts and $58 \%-63 \%$ to CE-chirp ${ }^{\circledR}$ LS. Thirdly, data also clearly show that click stimuli evoke larger CM amplitudes compared to $2 \mathrm{kHz}$ tone bursts and CE-chirp ${ }^{\circledR}$ LS (see Figure 3). In contrast to prevailing stimulation rates that are clinically applied to obtain APs and/or wave $\mathrm{V}$ of the auditory brainstem (typically below $15 \mathrm{~Hz}$ ), CMs do not require these lower stimulation rates since they do not depend on neural reactivity [1]. Although loss of auditory $\mathrm{AP}$ or peak $\mathrm{V}$ responses can occur by using a relatively high stimulation rate $(87.1 \mathrm{~Hz})$, the total measurement time can significantly be reduced, especially as clamped tube recordings are obligatory to confirm CMs [17]. In contrast to the two other sinusoidal stimuli, instantaneous fast activation of the basilar membrane in response to click stimuli enhance the CM amplitude, reflecting the total summation of the spatially activated OHC currents [3]. Since the power spectrum of a click stimulus mainly consists of relatively high frequencies, it is the basal part of the cochlea that is activated first [20], explaining the larger amplitudes due to the proximity of the recording TM electrode to the source [13].

It was also one of the aims of this study to analyze the surplus value of the chirp stimulus (see Appendix B) to evoke CMs. As far as we know, this is the only study using a TM-ECochG setup to obtain CM with chirps compared to conventional clicks and tone bursts. Although we are aware that the presentation level of the (shorter) click was higher in an absolute sense compared to the sinusoidal stimuli, based on the high success rates and the preceding loudness scaling task we performed in a pilot phase in order to equate the subjective loudness sensations of all three stimuli (following previous loudness scaling experiments investigating the effect of stimulus duration on loudness perception, e.g., [21]), the additional value of using a chirp stimulus seems to be limited, or at least not superior to a tone burst stimulus.

With respect to the differences between the sexes, the $\mathrm{CM}$ amplitudes of clicks were significantly larger for males than for females (Table 4). In contrast to the other two stimulus types, clicks showed larger standard deviations, regardless of the HPF setting. This inconsistency may be explained by the limited and low response amplitudes evoked by the other two stimuli, explaining the small standard deviation. Although both sexes showed larger standard deviations in CM amplitudes evoked by clicks, changing the HPF from 3.3 to $100 \mathrm{~Hz}$ only seems to affect males. However, in retrospect, it appeared that this was mainly due to data of two male subjects who showed extremely high CM amplitudes in $100 \mathrm{~Hz} \mathrm{HPF} \mathrm{condition.}$

With respect to the HPF, CM morphologies as well as success rates did not seem to be influenced by the choice for a high or low HPF setting ( $3.3 \mathrm{vs.} 100 \mathrm{~Hz}$ ). Based on the spectral content of the CE-chirp ${ }^{\circledR}$ LS stimulus (broadest frequency spectrum content), we expected that the difference between these two HPF might have some impact on the response morphology. In contrast, our data showed that the HPF did not really change the CMs for the BB-CE chirp, similar to the other two stimulus types. Although clinicians have been explicitly advocating for specific HPF settings in the past, e.g., $5 \mathrm{~Hz}[14,15]$ or $100 \mathrm{~Hz}[16,17]$, the present study did not show a clear preference for one of the two HPFs. This could be explained by the fact that (1) current EP-recording devices might have implemented higher quality filters, i.e., higher-order and more feasible modern digital filters, and (2) most of the previous ECochG studies were aimed at SP/AP potentials recordings instead of $\mathrm{CMs}$; the use of widening the bandpass filter of the preamplifier for conventional hearing threshold ECochGs was needed to enable recognition of the DC component (SP) as well as the AC component (AP) [1]. Using higher HPFs may therefore significantly influence $\mathrm{SP} / \mathrm{AP}$ recordings, but it may have less influence on CM morphology.

Additionally, to record CMs as part of the TM-ECochG, we also acquired auditory neural responses (SP/APs) at lower stimulation rates $(13.1 \mathrm{~Hz})$ to confirm a correct hardware setup and interpretation of all components (data not shown). Although we were only interested in CMs, we would like to mention a phenomenon that might be relevant for clinicians who intend to use TM-ECochG for SP/AP recordings in clinical practice. In some subjects, it was very remarkable that absolute AP latencies were prolonged in comparison to TM-ECochG outcomes reported by other TM-ECochG studies, such as Grasel et al. [19]. 
Since all our subjects had normal hearing, we assumed that this peak latency delay was caused by a conductive component due to the presence of (too much) residual xylocaine in the ear canal. It is known that when vibrations are poorly transmitted between two materials with different densities, such as air and water, this mismatch can lead to a loss of intensity [22]. Drying the ear canal during preparation was manually performed with a cotton bud, but it might be that in some subjects the cotton bud had absorbed less xylocaine fluid during ear canal cleaning before auditory stimulation than in others. Though APs were not our research goal, we have repeated ECochG in a few subjects with significantly prolonged AP latencies, considering insufficient absorption of xylocaine in the ear canal. These repeated measurement results, acutely obtained in the same session, indeed showed normal AP latencies after thorough xylocaine absorption (see Appendix A). Although this requires more additional research, based on these limited data, we suggest clinicians at least to control for xylocaine dose absorption before acquiring SP/APs with TM electrodes.

The present research shows that application of the less invasive TM electrode in ECochG is feasible to obtain consistent CMs in clinical settings. Thus, in contrast to SP/AP recordings, $\mathrm{CM}$ has the advantage in that it allows the use of faster stimulation rates (e.g., $87.1 \mathrm{~Hz}$, only limited by the length of stimuli) and, as a result, shorter measurement times. Clinical application of TM electrodes to record CMs might be limited to specific patient groups, but it has the advantage that it is less invasive compared to TT-ECochG. It has also been suggested that ET-ECochG might be a promising tool as the preoperative assessment in CI recipients because of its limited patient discomfort [10]. Future CM research should increase our knowledge of intracochlear structure preservation during cochlear implantation (CI), and hence, preoperative counseling or even side choice of implantation when structure preservation would show a substantial impact on postoperative hearing performance. Recent intraoperative CI studies already seem to point to a significant role of CM analyses [10]. Other applications of ET-ECochG are the assessment of patients suspected of ANSD [8] or of patients with mitochondrial disorders [7], with the goal to assess $\mathrm{OHC}$ functionality or the site of lesion.

In conclusion, this study contributed to the field of ECochG research with less invasive and more patient-friendly flexible silicon-shielded TM electrodes. The present study confirmed that this procedure can also be used to obtain CMs through efficient recording from the site of the tympanic membrane, provided that adequate preparation and experience of the clinician are met. Clicks generate the largest $\mathrm{CM}$ amplitude but, more importantly, show the highest success rate compared to the used sinusoidal stimuli and is therefore recommended as the preferred stimulus for confirming presence of CMs. The influence of using a low/high cut-off frequency of the HPF did not substantially change CM morphology. Stimulation and recording parameters for ET-ECochG are proposed to capture CMs with TM electrodes.

Author Contributions: Conceptualization, L.M.C. and A.J.B.; methodology, L.M.C. and A.J.B.; formal analysis, L.M.C. and A.J.B.; investigation, L.M.C. and A.J.B.; data curation, L.M.C. and A.J.B.; writingoriginal draft preparation, L.M.C. and A.J.B.; writing-review and editing, A.J.B.; supervision, A.J.B.; project administration, L.M.C. and A.J.B. All authors have read and agreed to the published version of the manuscript.

Funding: This research received no external funding.

Institutional Review Board Statement: The study was conducted according to the guidelines of the Declaration of Helsinki and approved by the Local Ethics Committee of the Radboud University Medical Centre Nijmegen (protocol NL69772.091.19/approval on 13-05-2019).

Informed Consent Statement: Informed consent was obtained from all subjects involved in the study.

Data Availability Statement: The datasets generated for this study are available on request to the corresponding author.

Acknowledgments: The authors want to acknowledge Jeroen Smits, for his assistance during otoscopy. 
Conflicts of Interest: The authors declare no conflict of interests or any funding from third parties or manufacturers of the products that have been used in this research.

\section{Appendix A}
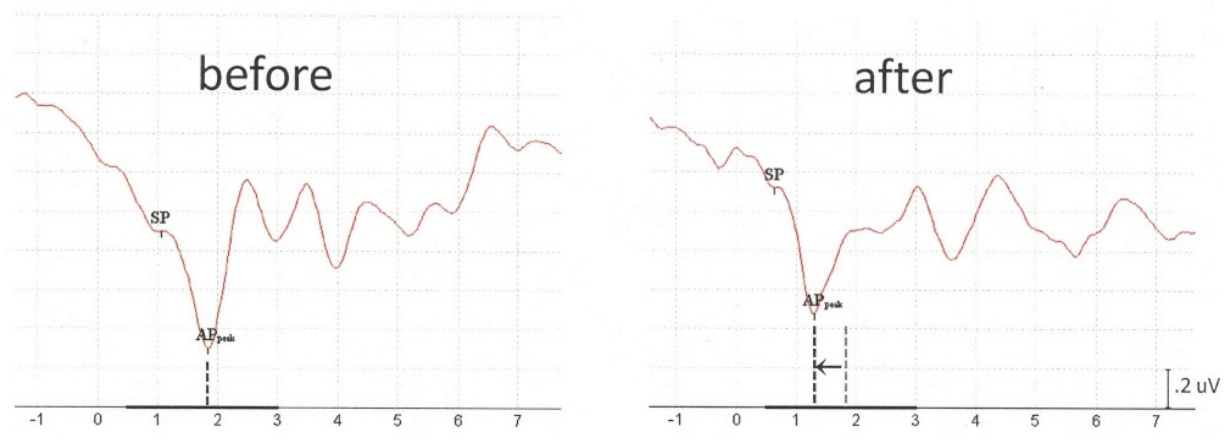

Figure A1. Typical SP / AP example of acute TM-ECochG within-subject recording before absorption of xylocaine showing a prolonged AP latency (left graph) and after absorption (right graph). Responses obtained by $90 \mathrm{dBnHL}$ click stimulus in alternating polarity, stimulation rate $11.3 / \mathrm{s}$ and band pass filtered $100-3000 \mathrm{~Hz}$.

\section{Appendix B}

For a description of chirp stimuli used in the present study, we refer to Elberling and Don [23]: the main principle of using the broad band chirp is to stimulate the basilar membrane simultaneously due to the presentation of a broad band stimulus that contains multi-frequencies [20,23]. Typically, chirp stimuli consist of multi-frequencies that are based on a model covering the traveling wave times in the cochlea. For the present study, a BB CE-chirp ${ }^{\circledR}$ LS was used that was designed so that the zero point on the time axis (t0) corresponds to the frequency of $10 \mathrm{kHz}$ in the underlying delay model. This point corresponds to the starting point ( $t 0)$ of the recording in the Interacoustics EP25 device, functioning as the temporal reference for every EP recording. Using this chirp, the $10 \mathrm{kHz}$ component is delayed by $1.5 \mathrm{~ms}$ to arrive at the start at $0 \mathrm{~ms}$, thus resulting in prolonged latencies of the chirp for all frequencies [24]. For other examples of chirp models, we would like to refer to the excellent work of Fobel and Dau [25], who gave an overview of different chirp models that have been developed. Chertoff et al. [20] have clearly described the temporal and spectral differences between clicks and chirps in time and frequency domain. They have reported that small differences in spectral contents were found between these two stimuli below $4 \mathrm{kHz}$ with the click stimulus having more energy than the chirp stimulus.

\section{References}

1. Ferraro, J.A.R.; Ruth, J.A. Electrocochleography. In Principles \& Applications in Auditory Evoked Potentials; Jacobson, J.T., Ed.; Allyn \& Bacon: Boston, MA, USA, 1994; pp. 101-122. ISBN 020-514-846-8.

2. Choudhury, B.; Fitzpatrick, D.C.; Buchman, C.A.; Wei, B.P.; Dillon, M.T.; He, S.; Adunka, O.F. Intraoperative round window recordings to acoustic stimuli from cochlear implant patients. Otol. Neurotol. 2012, 33, 1507-1515. [CrossRef] [PubMed]

3. Cheatham, M.A.; Naik, K.; Dallos, P. Using the cochlear microphonic as a tool to evaluate cochlear function in mouse models of hearing. J. Assoc. Res. Otolaryngol. 2011, 12, 113-125. [CrossRef] [PubMed]

4. Perlman, H.; Case, T. Electrical phenomena of the cochlea in man. Arch. Otol. 1941, 34, 710-718. [CrossRef]

5. Yoshie, N.; Ohashi, T.; Suzuki, T. Non-surgical recording of auditory nerve action potentials in man. Laryngoscope 1967, 77, 76-85. [CrossRef]

6. Lamounier, P.; Gobbo, D.A.; de Souza, T.S.A.; de Oliveira, C.A.C.P.; Bahmad, F., Jr. Electrocochleography for Ménière's disease: Is it reliable? Braz. Otorhinolaryngol. 2014, 80, 527-532. [CrossRef] [PubMed]

7. Chennupati, S.K.; Levi, J.; Loftus, P.; Jornlin, C.; Morlet, T.; O’Reilly, R.C. Hearing loss in children with mitochondrial disorders. Int. J. Pediatr. Otorhinolaryngol. 2011, 75, 1519-1524. [CrossRef] 
8. Starr, A.; Sininger, Y.; Nguyen, T.; Michalewski, H.J.; Oba, S.; Abdala, C. Cochlear receptor (microphonic and summating potentials, otoacoustic emissions) and auditory pathway (auditory brain stem potentials) activity in auditory neuropathy. Ear Hear 2001, 22, 91-99. [CrossRef]

9. Choudhury, B.; Adunka, O.F.; Demason, C.E.; Ahmad, F.I.; Buchman, C.A.; Fitzpatrick, D.C. Detection of intracochlear damage with cochlear implantation in a gerbil model of hearing loss. Otol. Neurotol. 2011, 32, 1370-1378. [CrossRef] [PubMed]

10. Trecca, E.M.C.; Riggs, W.J.; Mattingly, J.K.; Hiss, M.M.; Cassano, M.; Adunka, O.F. Electrocochleography and Cochlear Implantation: A Systematic Review. Otol. Neurotol. 2020, 41, 864-878. [CrossRef]

11. Zhang, M. Response pattern based on the amplitude of ear canal recorded cochlear microphonic waveforms across acoustic frequencies in normal hearing subjects. Trends Amplif. 2012, 16, 117-126. [CrossRef]

12. Soares, I.D.; Menezes, P.L.; Carnaúba, A.T.; de Andrade, K.C.; Lins, O.G. Study of cochlear microphonic potentials in auditory neuropathy. Braz. J. Otorhinolaryngol. 2016, 82, 722-736. [CrossRef] [PubMed]

13. Bonucci, A.S.; Hyppolito, M.A. Comparison of the use of tympanic and extratympanic electrodes for electrocochleography. Laryngoscope 2009, 119, 563-566. [CrossRef]

14. Campbell, K.C.; Harker, L.A.; Abbas, P.J. Interpretation of electrocochleography in Meniere's disease and normal subjects. Ann. Otol. Rhinol. 1992, 101, 496-500. [CrossRef]

15. Wuyts, F.L.; Van de Heyning, P.; Van Spaendonck, M.; Molenberghs, G. A review of electrocochleography: Instrumentation settings and meta-analysis of criteria for diagnosis of endolymphatic hydrops. Acta Otolaryngol. 1997, 117, 14-20. [CrossRef]

16. Shi, W.; Ji, F.; Lan, L.; Liang, S.-C.; Ding, H.-N.; Wang, H.; Li, N.; Li, Q.; Li, X.-Q.; Wang, Q.-J. Characteristics of cochlear microphonics in infants and young children with auditory neuropathy. Acta Otolaryngol. 2012, 132, 188-196. [CrossRef]

17. British Society of Audiology. Recommended Procedure Cochlear Microphonic Testing. Available online: https://www.thebsa. org.uk/wp-content/uploads /2019/01/FINAL-JAN2019-Recommended-Procedure-for-Cochlear-Microphonic-Testing-GL2101-19.pdf (accessed on 29 December 2020).

18. Redondo-Martínez, J.; Morant-Ventura, A.; Robledo-Aguayo, D.; Ayas-Montero, A.; Mencheta-Benet, E.; Marco-Algarra, J. Extra-tympanic electrocochleography in a normal population. A descriptive study. Acta Otorrinolaringol. Esp. 2016, 67, $254-260$. [CrossRef]

19. Grasel, S.S.; Beck, R.M.O.; Loureiro, R.S.C.; Rossi, A.C.; de Almeida, E.R.; Ferraro, J. Normative data for TM electrocochleography measures. J. Otol. 2017, 12, 68-73. [CrossRef] [PubMed]

20. Chertoff, M.; Lichtenhan, J.; Willis, M. Click-and chirp-evoked human compound action potentials. J. Acoust. Soc. Am. 2010, 127, 2992-2996. [CrossRef] [PubMed]

21. Moore, B.C.J. An introduction of the Psychology of Hearing, 5th ed.; Academic Press: Cambridge, MA, USA, 2003; ISBN 978-012-505-628-1.

22. Alberti, P.W. The anatomy and physiology of the ear and hearing. In Occupational Exposure to Noise: Evaluation, Prevention and Control; University of Toronto: Toronto, ON, Canada, 2001.

23. Elberling, C.; Don, M. A direct approach for the design of chirp stimuli used for the recording of auditory brainstem responses. J. Acoust. Soc. Am. 2010, 128, 2955-2964. [CrossRef] [PubMed]

24. Kristensen, S.G.B.; Elberling, C. Auditory Brainstem Responses to Level-Specific Chirps in Normal-Hearing Adults. J. Am. Acad. Audiol. 2012, 23, 712-721. [CrossRef]

25. Fobel, O.; Dau, T. Searching for the optimal stimulus eliciting auditory brainstem responses in humans. J. Acoust. Soc. Am. 2004, 116, 2213-2222. [CrossRef] [PubMed] 